\title{
Large mass dileptons from the passage of jets through quark gluon plasma
}

\author{
Dinesh K. Srivastava f and Charles Gale \\ Physics Department, McGill University, 3600 University Street, Montreal, H3A 2T8, Canada \\ Rainer J. Fries \\ Department of Physics, Duke University, Durham, NC 27708-0305, USA
}

(Dated: November 4, 2018)

\begin{abstract}
We calculate the emission of large mass dileptons originating from the annihilation of quark jets passing through quark gluon plasma. Considering central collisions of heavy nuclei at SPS, RHIC and LHC energies, we find that the yield due to the jet-plasma interaction gets progressively larger as the collision energy increases. We find it to be negligible at SPS energies, of the order of the Drell-Yan contribution and much larger than the normal thermal yield at RHIC energies and up to a factor of ten larger than the Drell-Yan contribution at LHC energies. An observation of this new dilepton source would confirm the occurrence of jet-plasma interactions and of conditions suitable for jet-quenching to take place.
\end{abstract}

\section{INTRODUCTION}

Collisions of relativistic heavy ions are performed with a hope to create a plasma of quarks and gluons (QGP) and to study the deconfined state of strongly interacting matter. Theoretical investigations of the formation, evolution and signatures of the QGP have played an important role in planning the experiments for these studies. One such investigation concerns the radiation of dileptons which are considered as penetrating probes of such matter due to their very long mean free path. Several sources of large mass dileptons have been identified and studied. These include the radiation of Drell-Yan pairs from the annihilation of primary partons in the nuclei [1], thermal dileptons from the annihilation of quarks and antiquarks in the plasma [2], and from hadronic reactions in the hot hadronic gas [3] following the hadronization of the plasma. Dileptons from the correlated decay of charm and bottom quarks have also been considered [ 四. Dileptons from the decay of hadrons like $\rho$ and $\phi$ will provide information about the possible medium modification of hadrons. The pre-equilibrium production of dileptons has also been investigated by several authors in various approximations [5, 6, 7, 8, 9.

Experimentally the low mass dilepton excess observed in the nucleus-nucleus collisions at SPS energies [10] has lead to suggestions that interactions among hadrons, and perhaps their properties as well, are altered in hot and dense nuclear matter [11]. The interpretation of the excess observed in intermediate mass dileptons 12 has been done in terms of a thermal hadronic environment with some quark-gluon plasma component $[13,14,15$. The measurement of dileptons has already made a considerable progress at RHIC experiments 16].

In the present work, we discuss the emission of large mass dileptons induced by the passage of high energy

\footnotetext{
*on leave from: Variable Energy Cyclotron Centre, 1/AF Bidhan Nagar, Kolkata 700 064, India
}

quark-jets through the QGP. The general formulation for their emission rate along with with a discussion of the evolution of the plasma, including the possible chemical equilibration, and the spectrum of jets and Drell-Yan dileptons, is given in Sect. II. The numerical results and a discussion are given in Sect. III followed by a summary.

\section{FORMULATION}

Let us consider a collision of two heavy nuclei at relativistic energies. A large number of minijets produced in the collision and the subsequent rescattering of the partons will lead to the formation of a thermalized plasma at some initial temperature $T_{0}$ at time $\tau_{0}$. This plasma may or may not be in chemical equilibrium.

The high energy partons, which are produced at times $\tau \sim 1 / p_{T} \ll \tau_{0}$, will traverse through this plasma, losing energy through collisions and radiation of gluons, a phenomenon widely known as jet-quenching and considered to be an important probe of the QGP [17].

The quark jets passing through the plasma will produce large mass dileptons by annihilation with the thermal antiquarks. They will also produce high energy photons 18. If these additional dileptons and photons are detected, they will provide a confirmation for the formation of the plasma and the occurrence of jet-plasma interactions and prepare the ground for a deeper understanding of the jet-quenching phenomenon and the evolution of the colliding system. We shall see that the production is quite sensitive to the temperatures at early times and controlled by the initial distribution of high energy partons.

In order to proceed we assume that the phase space distribution of the quarks in the medium can be decomposed as

$$
f(\mathbf{p})=f_{\mathrm{th}}(\mathbf{p})+f_{\text {jet }}(\mathbf{p})
$$

where we have a thermal component that is characterized 
by a temperature $T$,

$$
f_{\mathrm{th}}(\mathbf{p})=\exp (-E / T) .
$$

and a jet component which is assumed to be limited to partons having a $p_{T} \gg 1 \mathrm{GeV}$. This separation is kinematically reasonable, as the jet spectra fall off as a power law and can thus easily be differentiated from thermal components. We shall, somewhat arbitrarily, limit them to having $p_{T} \geq 4 \mathrm{GeV}$. In addition we give some results when only jets with $p_{T} \geq 6 \mathrm{GeV}$ are considered.

The phase-space distribution for the quark-jets is given by the perturbative QCD result for the jet yield as 19.

$$
\begin{aligned}
& f_{\text {jet }}(\mathbf{p})=\frac{1}{g_{q}} \frac{(2 \pi)^{3}}{\pi R_{\perp}^{2} \tau p_{T} \cosh y} \frac{d N_{\text {jet }}}{d^{2} p_{T} d y} \\
& \quad \times \delta(\eta-y) \Theta\left(\tau-\tau_{i}\right) \Theta\left(\tau_{\max }-\tau\right) \Theta\left(R_{\perp}-r\right) .
\end{aligned}
$$

where $g_{q}=2 \times 3$ is the spin and colour degeneracy of the quarks, $R_{\perp}$ is the transverse dimension of the system, $\tau_{i} \sim 1 / p_{T}$ is the formation time for the jet and $\eta$ is the space time rapidity. We shall take $\tau_{\max }$ as the smaller of the lifetime of the QGP $\left(\tau_{f}\right)$ and the time taken by the jet produced at position $\mathbf{r}$ with a velocity $\mathbf{v}$ in the transverse direction to reach the surface of the plasma. The distance covered by the parton during this passage is given by

$$
d=-r \cos \phi+\sqrt{R_{\perp}^{2}-r^{2} \sin ^{2} \phi}
$$

where $\cos \phi=\widehat{\mathbf{v}} \cdot \widehat{\mathbf{r}}$. We assume here that the parton is massless and travels with the speed of light. The boost invariant Bjorken correlation [20] between the rapidity $y$ and $\eta$ is assumed.

These phase space distributions can be folded with the cross section of the annihilation of the quarks to obtain the rate of emission of the dileptons. The rates are then convoluted with the space time history of the evolution of the plasma, using a standard procedure.

\section{A. Rate of emission of dileptons}

The cross section for quark-antiquark annihilation into dileptons $\left(a b \rightarrow \ell^{+} \ell^{-}\right)$is given by

$$
\sigma\left(M^{2}\right)=\frac{4 \pi}{3} \frac{\alpha^{2}}{M^{2}} N_{c}(2 s+1)^{2} \sum_{f} e_{f}^{2}
$$

where the sum runs over the flavour of quarks, $N_{c}=3$, and $s$ and $e_{f}$ stand for the spin and the charge of the quark.

Using kinetic theory the reaction rate for the above process can be written as

$$
R=\int \frac{d^{3} p_{a}}{(2 \pi)^{3}} f_{a}\left(\mathbf{p}_{a}\right) \int \frac{d^{3} p_{b}}{2 \pi^{3}} f_{b}\left(\mathbf{p}_{b}\right) \sigma\left(M^{2}\right) v_{\mathrm{rel}},
$$

where $f_{i}$ stands for the phase-space distribution of the quark or the antiquark, $\mathbf{p}_{a}$ and $\mathbf{p}_{b}$ are their momenta respectively and the relative velocity is

$$
v_{\mathrm{rel}}=\frac{E_{a} E_{b}-\mathbf{p}_{a} \mathbf{p}_{b}}{E_{a} E_{b}} .
$$

After some algebra [21] this can be rewritten as

$$
\frac{d R}{d M^{2}}=\frac{M^{6}}{2} \frac{\sigma\left(M^{2}\right)}{(2 \pi)^{6}} \int x_{a} d x_{a} d \phi_{a} x_{b} d x_{b} d \phi_{b} d y_{a} d y_{b} f_{a} f_{b} \delta\left[M^{2}-2 M^{2} x_{a} x_{b} \cosh \left(y_{a}-y_{b}\right)+2 M^{2} x_{a} x_{b} \cos \phi_{b}\right]
$$

where $x_{a}=p_{T}^{a} / M, x_{b}=p_{T}^{b} / M$ and $y_{a}$ and $y_{b}$ are the rapidities. The integrations over the azimuthal angles yield

$$
\begin{aligned}
\frac{d R}{d M^{2}}= & \frac{M^{4} \sigma\left(M^{2}\right)}{(2 \pi)^{5}} \int x_{a} d x_{a} x_{b} d x_{b} d y_{a} d y_{b} f_{a} f_{b} \\
& {\left[4 x_{a}^{2} x_{b}^{2}-\left\{2 x_{a} x_{b} \cosh \left(y_{a}-y_{b}\right)-1\right\}^{2}\right]^{-1 / 2} }
\end{aligned}
$$

such that,

$$
\begin{aligned}
-1 & \leq \frac{2 x_{a} x_{b} \cosh \left(y_{a}-y_{b}\right)-1}{2 x_{a} x_{b}} \leq 1, \\
0 & \leq x_{a, b} \leq \infty \\
-\infty & \leq y_{a, b} \leq \infty .
\end{aligned}
$$

When $f_{a}$ and $f_{b}$ are given by a thermal distribution

$$
f_{\text {th }}(\mathbf{p})=\exp (-E / T)=\exp \left(-p_{T} \cosh y / T\right)
$$

the above integral can be performed 22] to obtain

$$
\frac{d R}{d M^{2}}=\frac{\sigma\left(M^{2}\right)}{2(2 \pi)^{4}} M^{3} T K_{1}(M / T)
$$

The expression in Eq. (9) can be used for phase space distributions of arbitrary form. When the distributions $f_{a}$ and $f_{b}$ are taken as thermal, we shall call the yield as thermal and when one of them is thermal and the other one is from a jet, we call the yield as due to the passage of jets through the plasma in an obvious nomenclature. Noting that the jet-distribution (Eq. 3) depends on the quark flavour, we take charge-weighted average of 


\begin{tabular}{||c||c|c|c|c|c||}
\hline \hline & $\begin{array}{c}\tau_{0} \\
(\mathrm{fm} / c)\end{array}$ & $\begin{array}{c}T_{0} \\
(\mathrm{GeV})\end{array}$ & $\begin{array}{c}\lambda_{g}^{(i)} \\
-\end{array}$ & $\begin{array}{c}\lambda_{q}^{(i)} \\
-\end{array}$ & $\begin{array}{c}\epsilon_{i} \\
\left(\mathrm{GeV} / \mathrm{fm}^{3}\right)\end{array}$ \\
\hline \hline \multicolumn{6}{|c||}{ Bjorken formula } \\
\hline SPS & 0.20 & 0.345 & 1.0 & 1.0 & 26.10 \\
& 0.50 & 0.254 & 1.0 & 1.0 & 7.94 \\
\hline RHIC & 0.15 & 0.447 & 1.0 & 1.0 & 72.38 \\
& 0.50 & 0.297 & 1.0 & 1.0 & 14.43 \\
\hline LHC & 0.073 & 0.897 & 1.0 & 1.0 & 1172 \\
& 0.50 & 0.473 & 1.0 & 1.0 & 90.92 \\
\hline \multicolumn{6}{|c||}{ Self-screened parton cascade } \\
\hline RHIC & 0.25 & 0.67 & 0.34 & 0.064 & 61.4 \\
\hline LHC & 0.25 & 1.02 & 0.43 & 0.082 & 425 \\
\hline \hline
\end{tabular}

TABLE I: Initial conditions for the hydrodynamical expansion estimated from multiplicity densities using the Bjorken formula and from a self-screened parton cascade model 26.

the distributions for $u, d$, and $s$ quark-jets and for the corresponding anti-quark jets, while using $f_{\text {jet }}$ in Eq. (9):

$$
f_{j e t}^{q(\bar{q})}(\mathbf{p})=\sum_{f} e_{f}^{2} f_{\mathrm{jet}}^{f}(\mathbf{p}) / \sum_{f} e_{f}^{2}
$$

where the sum runs over the flavours of quarks (antiquarks). The thermal distributions are assumed to be flavour-independent, so that the relation (Eq. 11) is recovered for them.

\section{B. Evolution of the plasma}

The radiation of dileptons from an expanding and cooling plasma including the contributions from a mixed phase and the hadronic phase with transverse expansion of the plasma has been studied in great detail (see e.g. [15]). We are interested in the emission of dileptons having large masses and thus it is sufficient to consider emissions from the QGP phase. We also ignore the transverse expansion of the plasma [9].

As a first step, we assume that a thermally and chemically equilibrated plasma is produced in the collision at time $\tau_{0}$. Assuming furthermore an isentropic expansion [20, 23. one can write that

$$
\frac{2 \pi^{4}}{45 \zeta(3)} \frac{1}{A_{\perp}} \frac{d N}{d y}=4 a T_{0}^{3} \tau_{0}
$$

where $d N / d y$ is the particle rapidity density for the collision and $a=42.25 \pi^{2} / 90$ for a plasma of massless $u, d$ and $s$ quarks and gluons. We have taken the number of quark flavours as $\approx 2.5$ to account for the mass of $s$ quarks while evaluating $a$. The transverse area of the system is taken as $A_{\perp}=\pi R_{\perp}^{2}$ where $R_{\perp}=1.2 A^{1 / 3}$ is the transverse radius of the system for a head-on collision. We assume a rapid thermalization limited by $\tau_{0} \sim 1 / 3 T_{0}$ [21].
We take the particle rapidity density as 750 for central collisions of lead nuclei at SPS energies. At RHIC energies we estimate the particle rapidity density as $\approx 1260$, based on the measured charged particle pseudo-rapidity density for central collisions of gold nuclei at $\sqrt{s_{N N}}=$ $200 \mathrm{GeV}$. For central collisions of lead nuclei at LHC energies we use $d N / d y=5625$, as suggested in [21].

The initial temperatures and times from these estimates are given in Table $\mathbb{1}$ under the heading Bjorken formula. We also use an alternative estimate where the formation time is taken as $0.50 \mathrm{fm} / c$, so that the corresponding initial temperatures are considerably reduced. We shall see that this drastically alters the yield of dileptons from interactions within the plasma, while the yield due to the jet-plasma interaction only reduces by a factor of about 2 .

We further approximate the nuclei as being spherical in shape with a uniform density and assume that the energy density at the transverse position $r$ is proportional to the number of collisions so that

$$
\epsilon\left(\tau_{0}, r\right) \propto 4 \rho_{0}^{2}\left[R_{\perp}^{2}-r^{2}\right]
$$

where $\rho_{0}$ is the density of the nucleons. The normalization is then determined by the condition that

$$
A_{\perp} \epsilon_{0}=\int 2 \pi r \epsilon(r) d r
$$

where $\epsilon_{0}$ is the average energy density decided by the temperature $T_{0}$. We note that this results in a transverse profile for the initial temperature as

$$
T(r)=T_{0}\left[2\left(1-\frac{r^{2}}{R_{\perp}^{2}}\right)\right]^{1 / 4} .
$$

We account for the transverse profile of the jet production as well by introducing a factor $2\left(1-r^{2} / R_{\perp}^{2}\right)$ in expression (3) while performing the space-time integration

$$
d^{4} x=\tau d \tau r d r d \eta d \phi .
$$

The limits of the $\tau$-integration are $\left[\tau_{0}, \tau_{f}\right]$ for the purely thermal contribution. While estimating the yield of dileptons due to the passage of jets through the plasma the upper limit has to be taken as the minimum of $d / c$ and $\tau_{f}$. Assuming a Bjorken cooling (for the chemically equilibrated plasma), so that $T^{3} \tau$ is constant, we estimate $\tau_{f}$ to be

$$
\tau_{f}=\left[\frac{T_{0}}{T_{c}}\right]^{3} \tau_{0},
$$

so that it is about $2 \mathrm{fm} / c$ at SPS energies, about $3.3 \mathrm{fm} / c$ at RHIC energies, and about $13 \mathrm{fm} / c$ at LHC energies, when we take $T_{c}$ as $160 \mathrm{MeV}$, and $T_{0}$ and $\tau_{0}$ from Table 1 . Thus we note that the average value for $d$ 


\begin{tabular}{|c|c|c|c|c|c|}
\hline & & $C \quad\left[1 / \mathrm{GeV}^{2}\right]$ & $\begin{array}{ll}B & {[\mathrm{GeV}]}\end{array}$ & $\bar{\beta}$ & $\delta$ \\
\hline \multirow{6}{*}{ SPS } & $\mathrm{u}$ & $5.770 \times 10^{5}$ & 0.2657 & 6.638 & 7.682 \\
\hline & $\mathrm{d}$ & $4.508 \times 10^{5}$ & 0.2904 & 6.703 & 7.631 \\
\hline & $\mathrm{s}$ & $2.760 \times 10^{5}$ & 0.3276 & 6.846 & 10.696 \\
\hline & $\overline{\mathrm{u}}$ & $1.147 \times 10^{5}$ & 0.2826 & 6.781 & 10.949 \\
\hline & $\overline{\mathrm{d}}$ & $6.028 \times 10^{5}$ & 0.1809 & 6.402 & 10.942 \\
\hline & $\mathrm{g}$ & $3.180 \times 10^{5}$ & 0.5344 & 8.071 & 8.964 \\
\hline \multirow{6}{*}{ RHIC } & $\mathrm{u}$ & $9.113 \times 10^{2}$ & 1.459 & 7.679 & - \\
\hline & $\bar{d}$ & $9.596 \times 10^{2}$ & 1.467 & 7.662 & - \\
\hline & $\mathrm{s}$ & $1.038 \times 10^{2}$ & 1.868 & 8.642 & - \\
\hline & $\overline{\mathrm{u}}$ & $2.031 \times 10^{2}$ & 1.767 & 8.546 & - \\
\hline & $\overline{\mathrm{d}}$ & $2.013 \times 10^{2}$ & 1.759 & 8.566 & - \\
\hline & $\mathrm{g}$ & $4.455 \times 10^{3}$ & 1.7694 & 8.610 & - \\
\hline \multirow{6}{*}{$\mathrm{LHC}$} & $\mathrm{u}$ & $2.209 \times 10^{4}$ & 0.5635 & 5.240 & - \\
\hline & $\mathrm{d}$ & $2.493 \times 10^{4}$ & 0.5522 & 5.223 & - \\
\hline & $\mathrm{s}$ & $1.662 \times 10^{3}$ & 0.9064 & 5.548 & - \\
\hline & $\overline{\bar{u}}$ & $4.581 \times 10^{3}$ & 0.7248 & 5.437 & - \\
\hline & $\overline{\mathrm{d}}$ & $4.317 \times 10^{3}$ & 0.7343 & 5.448 & - \\
\hline & $\mathrm{g}$ & $1.229 \times 10^{5}$ & 0.7717 & 5.600 & - \\
\hline
\end{tabular}

TABLE II: Parameters for the minijet distribution $d N / d^{2} p_{T} d y$ given in Eq. (31) at $y=0$ for $\mathrm{Pb}+\mathrm{Pb}$ at $\sqrt{s_{\mathrm{NN}}}=17.4 \mathrm{GeV}$ (SPS), $\mathrm{Au}+\mathrm{Au}$ at $\sqrt{s_{\mathrm{NN}}}=200 \mathrm{GeV}(\mathrm{RHIC})$ and for $\mathrm{Pb}+\mathrm{Pb}$ at $\sqrt{s_{\mathrm{NN}}}=5.5 \mathrm{TeV}$ (LHC). For all parameterizations $K=2.5$. CTEQ5L plus EKS98 nuclear parton distributions are used. The range of validity is $p_{T}=2-20 \mathrm{GeV}$ for RHIC and LHC and $2-7 \mathrm{GeV}$ for SPS.

$$
\begin{aligned}
\langle d\rangle & =\frac{3}{2 \pi R_{\perp}} \int_{0}^{2 \pi} d \phi \int_{0}^{R_{\perp}} d r d\left(1-r^{2} / R_{\perp}^{2}\right) \\
& =\frac{R_{\perp}}{2}\left[3{ }_{3} F_{2}\left(-\frac{1}{2}, \frac{1}{2}, \frac{1}{2} ; 1, \frac{3}{2} ; 1\right)-{ }_{3} F_{2}\left(-\frac{1}{2}, \frac{1}{2}, \frac{1}{2} ; 1, \frac{5}{2} ; 1\right)\right] \approx 0.94 R_{\perp}
\end{aligned}
$$

is smaller than the lifetime of the plasma only at LHC energies. In the above, the ${ }_{p} F_{q}$ 's are generalized hypergeometric functions. Of course the transverse expansion of the plasma, which should be seen at LHC will reduce this lifetime [9, 24 to some extent. Thus, on an average, the jet covers only a short distance in the hot plasma, before the medium cools down to $T_{c}$ [25], except at LHC, where the jets get to traverse the entire length of the system, while it is still hot and deconfined. We shall see that this leads to a large yield of dileptons from the jet-plasma interaction at LHC.

\section{Chemically equilibrating plasma}

Several model calculations have suggested (see e.g. Ref. 26]) that while the plasma to be created in relativistic heavy ion collisions may attain kinetic equilibrium fairly quickly, the evolution to the chemical equilibrium
27. proceeds only slowly through reactions of the type $g g \leftrightarrow q \bar{q}$ and gluon multiplication and fusion $g g \leftrightarrow g g g$. Defining the quark and gluon fugacities through

$$
n_{g}=\lambda_{g} \tilde{n}_{g}, n_{q}=\lambda_{q} \tilde{n}_{q},
$$

where $n_{i}$ are the actual densities and $\tilde{n}_{i}$ are the equilibrium densities for the parton species $i$, we can write

$$
\begin{aligned}
& \tilde{n}_{g}=\frac{16}{\pi^{2}} \zeta(3) T^{3}=a_{1} T^{3}, \\
& \tilde{n}_{q}=\frac{9}{2 \pi^{2}} \zeta(3) N_{f} T^{3}=b_{1} T^{3} .
\end{aligned}
$$

The energy density and the pressure of the plasma are then given by,

$$
\epsilon=3 P=\left[a_{2} \lambda_{g}+b_{2}\left(\lambda_{q}+\lambda_{\bar{q}}\right)\right] T^{4},
$$

where $a_{2}=8 \pi^{2} / 15, b_{2}=7 \pi^{2} N_{f} / 40$ and $N_{f} \approx 2.5$ is the number of dynamical quark flavours. We further assume 
that $\lambda_{q}=\lambda_{\bar{q}}$. Assuming a boost invariant longitudinal expansion, the relevant master equations can be solved to give

$$
\begin{aligned}
& \frac{1}{\lambda_{g}} \frac{d \lambda_{g}}{d \tau}+\frac{3}{T} \frac{d T}{d \tau}+\frac{1}{\tau}=R_{3}\left(1-\lambda_{g}\right)-2 R_{2}\left(1-\frac{\lambda_{q} \lambda_{\bar{q}}}{\lambda_{g}^{2}}\right), \\
& \frac{1}{\lambda_{q}} \frac{d \lambda_{q}}{d \tau}+\frac{3}{T} \frac{d T}{d \tau}+\frac{1}{\tau}=R_{2} \frac{a_{1}}{b_{1}}\left(\frac{\lambda_{g}}{\lambda_{q}}-\frac{\lambda_{\bar{q}}}{\lambda_{g}}\right),
\end{aligned}
$$

where the rate constants

$$
\begin{aligned}
& R_{2} \approx 0.24 N_{f} \alpha_{s}^{2} \lambda_{g} T \ln \left(1.65 / \alpha_{s} \lambda_{g}\right), \\
& R_{3}=1.24 \alpha_{s}^{2} T\left(2 \lambda_{g}-\lambda_{g}^{2}\right)^{1 / 2},
\end{aligned}
$$

are taken from Ref. [27] and include the effects of colour Debye screening and Landau-Pomeranchuk-Migdal suppression of the induced gluonic radiations. The initial conditions necessary for a numerical solution of the above equation are taken from the self screened parton cascade model [26] and given in Table If for the sake of completeness.

\section{Jets and Drell-Yan}

We estimate the jet production and Drell-Yan production of dileptons in lowest order pQCD. The jet cross section, normalized to one nucleon pair, for the production of partons (quarks, antiquarks or gluons) in primary hard interactions between partons $a$ and $b$ from different nuclei $A$ and $B$ is given by 28

$$
\begin{aligned}
\frac{d \sigma^{\mathrm{jet}}}{d^{2} p_{T} d y}=\sum_{a+b \rightarrow \mathrm{jet}} & \int_{x_{a}^{\min }}^{1} d x_{a} f_{a}^{A}\left(x_{a}\right) f_{b}^{B}\left(x_{b}\right) \\
& \times \frac{x_{a} x_{b} \sqrt{s_{\mathrm{NN}}}}{x_{a} \sqrt{s_{\mathrm{NN}}}-p_{T} e^{y}} \frac{1}{\pi} \frac{d \sigma_{a+b \rightarrow \mathrm{jet}}}{d t} .
\end{aligned}
$$

Here $f_{a}^{A}$ and $f_{b}^{B}$ are parton distributions for the colliding nuclei, so that

$$
\begin{aligned}
f_{a}^{A}\left(x, Q^{2}\right)=R_{a}^{A}\left(x, Q^{2}\right) \\
\times\left[\frac{Z}{A} f_{a}^{p}\left(x, Q^{2}\right)+\frac{N}{A} f_{a}^{n}\left(x, Q^{2}\right)\right],
\end{aligned}
$$

where $R_{a}^{A}$ is the nuclear modification of the structure function, $Z$ is the number of protons, $N$ is the number of neutrons and $Q^{2}=p_{T}^{2}$. The sum runs over all parton processes where a parton, forming a jet, can be produced from $a$ and $b$ with differential cross section $d \sigma / d t . \sqrt{s_{\mathrm{NN}}}$ is the center of mass energy of the colliding nuclei per nucleon pair, so that $s=x_{a} x_{b} s_{\mathrm{NN}}$. Furthermore we have

$$
\begin{aligned}
x_{b} & =\frac{x_{a} p_{T} e^{-y}}{x_{a} \sqrt{s_{\mathrm{NN}}}-p_{T} e^{y}}, \\
x_{a}^{\min } & =\frac{p_{T} e^{y}}{\sqrt{s_{\mathrm{NN}}}-p_{T} e^{-y}} .
\end{aligned}
$$
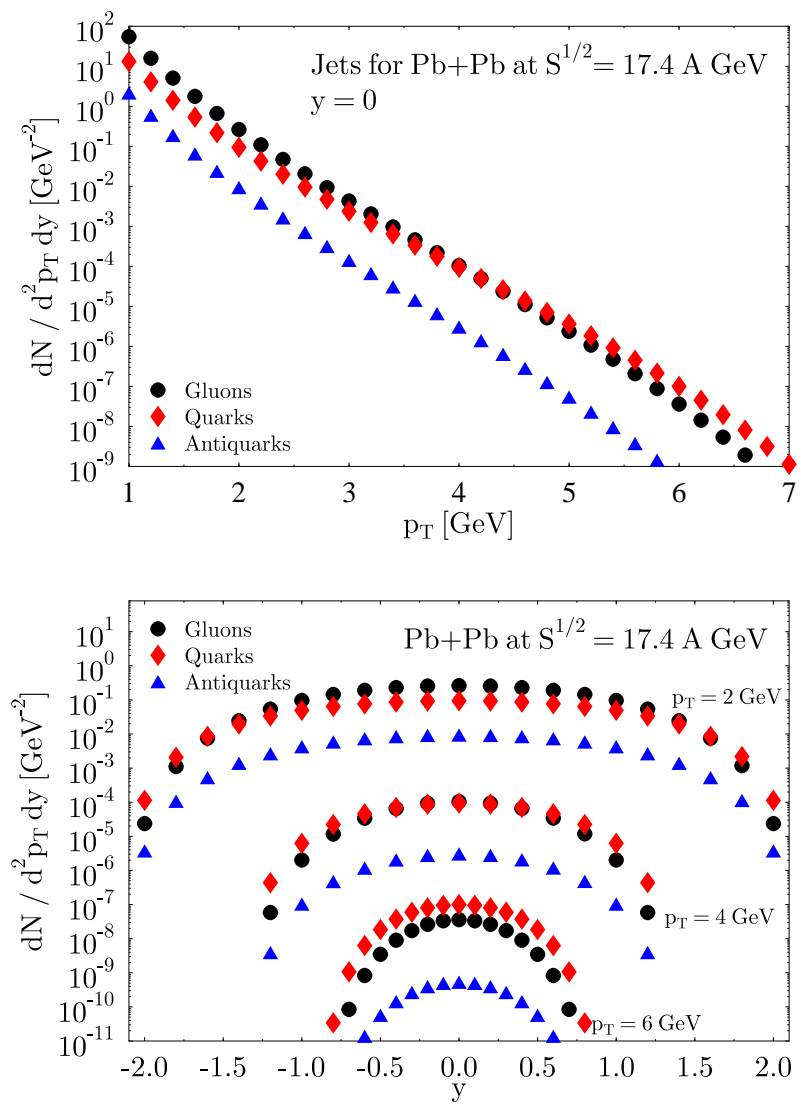

FIG. 1: Upper part: transverse momentum distribution of gluons, quarks and antiquarks at $y=0$ for central $\mathrm{Pb}+\mathrm{Pb}$ collisions at $\sqrt{s_{N N}}=17.4 \mathrm{GeV}$ at SPS. Points are calculated, the lines are drawn as a guide to the eye. Lower part: the rapidity distribution for gluons, quarks and antiquarks at SPS for $p_{T}=2,4$ and $6 \mathrm{GeV}$ (from top to bottom). In this, and in the following two figures, we have plotted the average of the three lightest flavours, $q=(u+d+s) / 3$, and $\bar{q}=(\bar{u}+\bar{d}+\bar{s}) / 3$, to avoid overcrowding.

We have used the CTEQ5L parton distributions 29] and EKS98 nuclear modifications [30. We further use a $K$-factor of 2.5 in the jet production to account for higher order corrections (see later). The results of our calculations can be found in Figs. 1, 2 and 3. We also give a fairly accurate parameterization for the results at RHIC and LHC energies;

$$
\begin{aligned}
\left.\frac{d N^{\mathrm{jet}}}{d^{2} p_{T} d y}\right|_{y=0} & =\left.T_{A A} \frac{d \sigma^{\mathrm{jet}}}{d^{2} p_{T} d y}\right|_{y=0} \\
& =K \frac{C}{\left(1+p_{T} / B\right)^{\beta}}
\end{aligned}
$$

for the number of quarks, antiquarks and gluons at $y=0$. For SPS it is necessary to include a multiplicative term $\left(1-p_{T} / 8.7 \mathrm{GeV}\right)^{\delta}$ to the right hand side of Eq. (31) in order to describe the $p_{T}$-spectrum close to the kinematic 

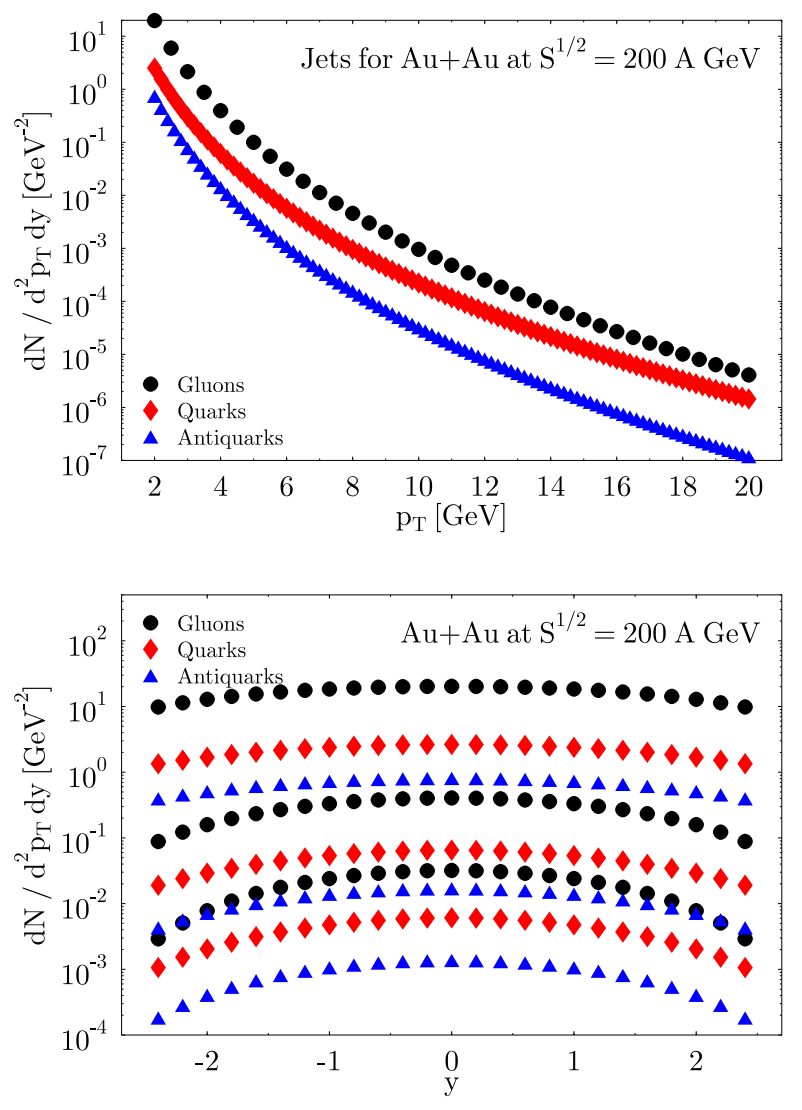

FIG. 2: Same as Fig. 1 for central $\mathrm{Au}+\mathrm{Au}$ at $\sqrt{s_{N N}}=200$ $\mathrm{GeV}$ at RHIC.

limit of $8.7 \mathrm{GeV}$. Here $T_{A A}=9 A^{2} / 8 \pi R_{\perp}^{2}$ is the nuclear thickness for zero impact parameter. Values for the parameters $B, C, \beta$ and $\delta$ can be found in Table II. We note that the rapidity distributions of the partons are quite flat near $y=0$ at RHIC and LHC energies. We have numerically verified that assuming a flat rapidity spectrum at SPS energies introduces an error only of the order of $10-20 \%$ as the expressions favour contributions from rapidities near $y=0$. In any case the yield at SPS energies is very low.

The cross-section for the Drell-Yan process is given by,

$$
\begin{array}{r}
\frac{d \sigma}{d M^{2} d y}=\frac{4 \pi \alpha^{2}}{9 M^{4}} \sum_{q} e_{q}^{2}\left[x_{1} f_{q}^{A}\left(x_{1}, M^{2}\right) x_{2} f_{\bar{q}}^{B}\left(x_{2}, M^{2}\right)\right. \\
+(q \leftrightarrow \bar{q})]
\end{array}
$$

Here the sum runs again over the quark flavours, and

$$
\begin{aligned}
& x_{1}=M e^{y} / \sqrt{s_{N N}}, \\
& x_{2}=M e^{-y} / \sqrt{s_{N N}} .
\end{aligned}
$$

These are scaled by $T_{A A}$ similar to Eq. (31) to obtain $d N / d M^{2} d y$ for the Drell-Yan production of dileptons.
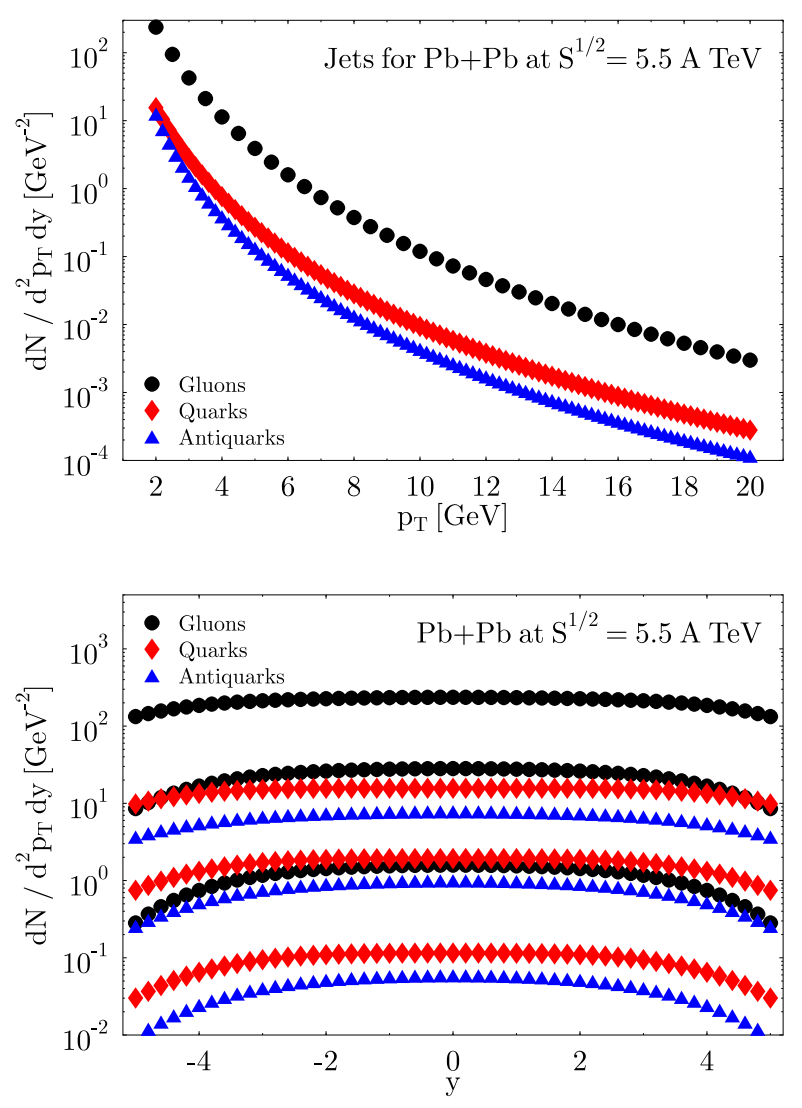

FIG. 3: Same as Fig. 11 for central $\mathrm{Pb}+\mathrm{Pb}$ at $\sqrt{s_{N N}}=5.5$ $\mathrm{TeV}$ at LHC.

\section{RESULTS AND DISCUSSION}

In Figs. 4 , 5 and 6 we plot the results for thermal dileptons, dileptons from the Drell-Yan process, and the dileptons from the passage of quark jets through the plasma for SPS, RHIC and LHC respectively. Note that gluon jets will contribute only at higher order.

At SPS energies, we recover (Fig. 1) the well known result that the large mass dileptons have their origin predominantly in the Drell-Yan process. Increasing the formation time from $0.20 \mathrm{fm} / c$ to $0.50 \mathrm{fm} / c$ - and thus lowering the initial temperature by $100 \mathrm{MeV}$ - drastically alters the thermal production (from the dash-dotted curve to the long-dashed one) while the yield from the proposed jet-plasma interaction, even though essentially negligible, is reduced by a factor of $\approx 2$ (from the solid line to the long-dashed one).

The jet-plasma interaction starts playing an interesting role at RHIC energies (Fig. 5), as now the corresponding yield is about only one third of the Drell-Yan contribution, and is much larger than the thermal contribution. Again lowering the initial temperature (now by about $150 \mathrm{MeV}$ ) by increasing the formation time to $0.50 \mathrm{fm} / c$ further enhances the importance of the yield 


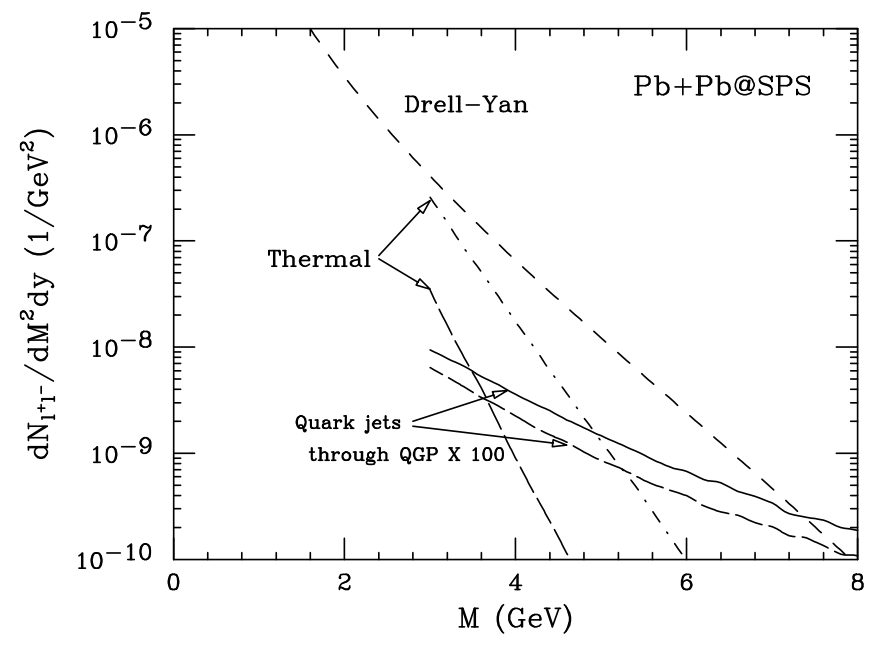

FIG. 4: Dilepton spectrum for $\mathrm{Pb}+\mathrm{Pb}$ at $\sqrt{s_{\mathrm{NN}}}=17.4 \mathrm{GeV}$ at SPS. The long dashed curves give the results when the formation time $\tau_{0}$ is raised to $0.50 \mathrm{fm} / c$, thus lowering the temperature.

due to jet-plasma interaction. We add that this production is of the same order as that attributed to secondarysecondary quark-antiquark annihilation in a dilepton production calculation done using an earlier version of the parton cascade model [6].

The much larger initial temperatures likely to be attained at the LHC and the much larger (mini)jet production lead to an excess of large mass dileptons from jet-plasma interactions which can be an order of magnitude larger (at $M=10 \mathrm{GeV}$ ) than that due to the DrellYan process. Again, reducing the initial temperature by raising the formation time to $0.50 \mathrm{fm} / c$ reduces the jetplasma yield by about a factor of 2 while the thermal yield is reduced far more. We recall that at LHC energies several calculations (see e.g. [4], 9, 21]) have reported a thermal yield larger than the Drell-Yan production. We find that the jet-plasma interaction enhances the large mass dilepton production considerably.

The equilibrating plasma scenario discussed suggests that, while the quark and gluon fugacities may initially be less than unity, the initial temperature could be larger (see Table 1). This results (see Fig. đ) in a situation where the jet-plasma yield remains large. It is larger than the thermal yield, but still smaller than the DrellYan contribution at RHIC energies.

The results at LHC energies (see Fig. 8) are particularly interesting because the large mass yield is completely dominated by the jet-plasma interactions, and this remains true even when only jets having $p_{T}>6$ $\mathrm{GeV}$ are considered.

We see that the quark jets passing through the QGP give rise to a large production of large mass dileptons, akin to the production of high energy photons through the same mechanism suggested earlier [18]. They should be absent in $p p$ collisions and if no QGP is formed. We

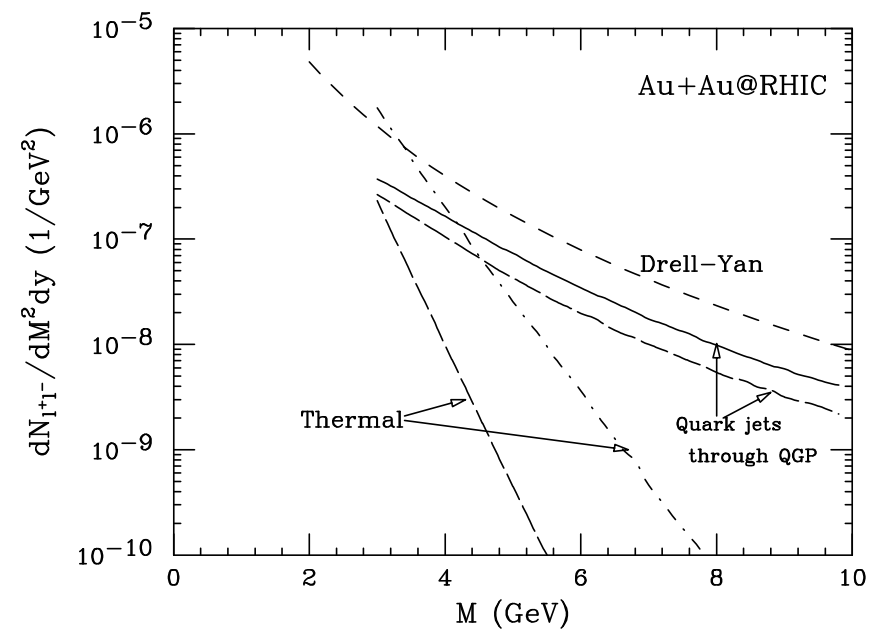

FIG. 5: Same as Fig. 4 for central $\mathrm{Au}+\mathrm{Au}$ at $\sqrt{s_{N N}}=200$ $\mathrm{GeV}$ at RHIC.

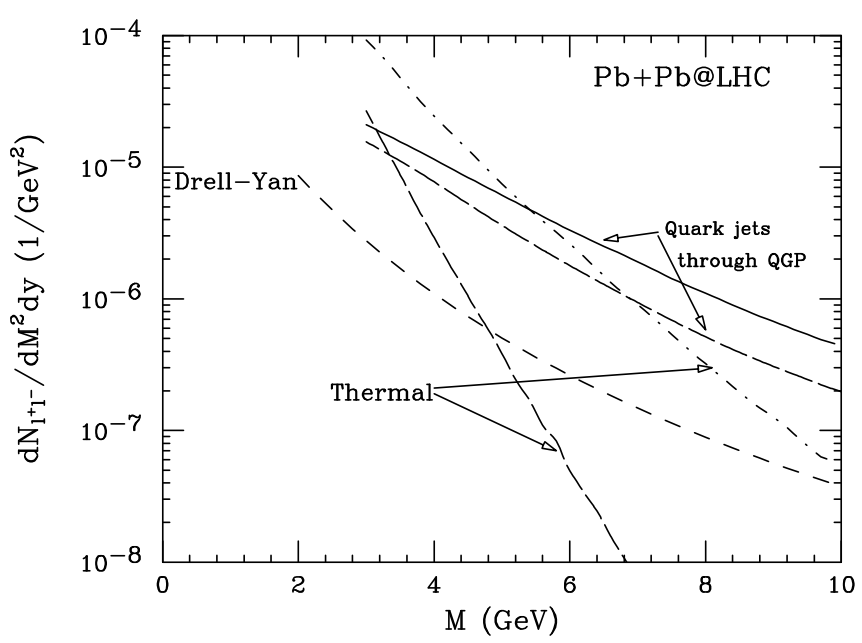

FIG. 6: Same as Fig. 1 for central $\mathrm{Pb}+\mathrm{Pb}$ at $\sqrt{s_{N N}}=5.5$ $\mathrm{TeV}$ at LHC.

have noted that the results are sensitive to the initial conditions and the time the jet spends in the medium.

Before summarizing, we would like to discuss some interesting extensions of this work. We have concentrated on quark jets. Gluon jets would give rise to dileptons through the process $q g \rightarrow q \gamma^{*}$. However, phase space considerations would tend to disfavour this process with respect to the channel considered in this work. But this contribution may still be large, especially as the gluonic jets are more numerous. This could also compensate for the additional $\alpha_{s}$ needed. However, a previous dynamical calculation did find this contribution to be sub-leading [6]. The discussions in this work can be easily extended to diphotons [31. Connected to that is the interesting question of the transverse momentum spectrum of the dileptons, both from the Drell-Yan process and the jetplasma interaction. No K-factors were used for the elec- 
tromagnetic processes in this first baseline investigation. A detailed quantitative calculation will of course require those, and we shall report on the results of those investigations in a future publication [32]. We also add that one may in principle have a energy and $p_{T}$ dependent $K$-factor [33] while estimating the jet-distributions. However, it is known that at higher energies, where the discussed process becomes important, the $p_{T}$-dependence of the $K$-factor is weak [33], when comparing NLO contributions to the LO contributions. The precise value for the K-factor is thus only a constant multiplicative factor and results for a different value can be easily obtained.

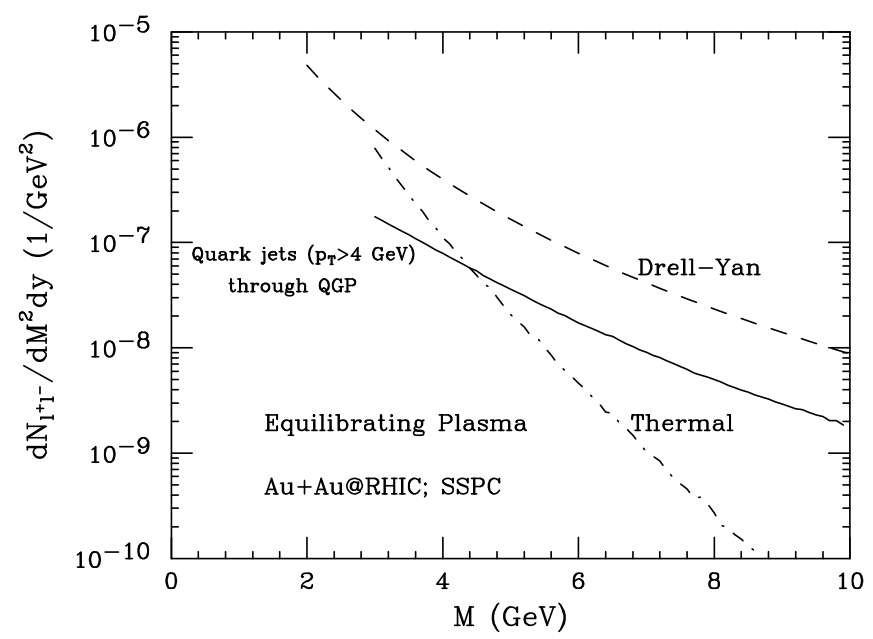

FIG. 7: Dilepton yield for central $\mathrm{Au}+\mathrm{Au}$ at $\sqrt{s_{N N}}=200$ $\mathrm{GeV}$ at RHIC assuming a chemically equilibrating plasma.

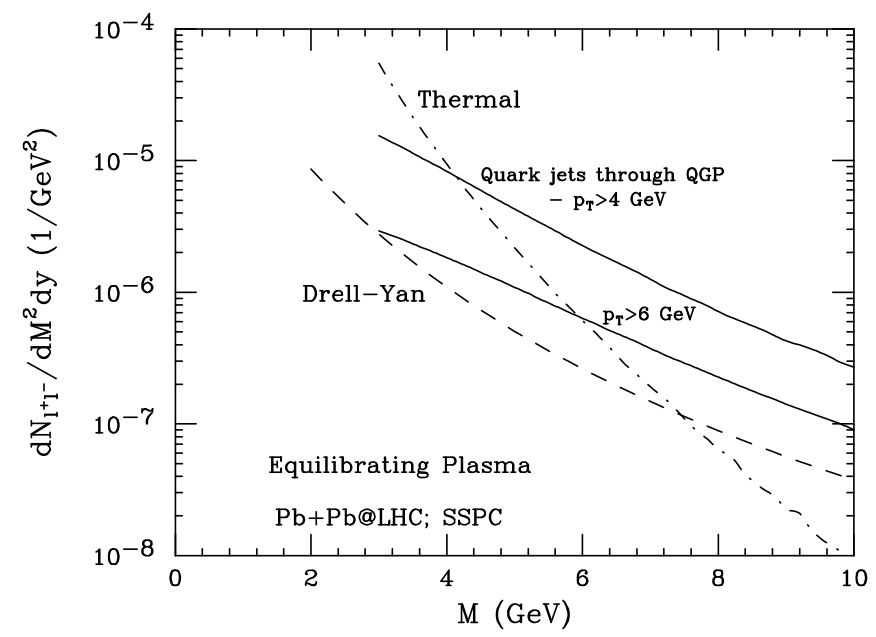

FIG. 8: Same as Fig. 月 for central $\mathrm{Pb}+\mathrm{Pb}$ at $\sqrt{s_{N N}}=5.5$ $\mathrm{TeV}$ at LHC.

A more serious modification of our results is expected from the fact that jets passing through the QGP will loose energy. We note though that quark jets, the focus of the present work, lose less energy than the gluon jets. Furthermore, the number of quarks in the medium having a given transverse momentum $p_{T}$ drops rapidly as time passes, mainly due to the cooling (in the plasma) and also due to the free streaming of the jets. This limits the production of the dileptons mostly to the early times. Several authors (see e.g., Ref. [34]) have discussed the effect of energy loss on the Drell-Yan production of dileptons. This can be easily incorporated in the treatment given here.

We have not compared our results to the correlated decay of charm quarks, which provide a large contribution to dileptons at RHIC and LHC energies [4], if the energy loss likely to be suffered by the charm quarks due to collisions and radiations is ignored. If we assume that the charm quarks may lose energy due to these processes [35], then these contributions will be suppressed [36]. This debate [37] has however not yet reached closure. A direct measurement could go a long way in settling this issue 38.

We also recall that the source of dileptons discussed in the present work corresponds to the so-called secondarysecondary interactions in the parton cascade model, discussed in Ref. [6]. However, it was pointed out [39] that the procedure adopted in Ref. [6] did not account for the fact that the final state partons emerging from a partonparton collision continue to participate in the development of the cascade by fragmenting and scattering while a virtual photon leaves the system. It will be worthwhile to calculate the dilepton production within a (correctly implemented) parton cascade model [40] with these considerations.

In brief, we have discussed a unique source of large mass dileptons in relativistic collisions of heavy nuclei. It arises from the passage of quark jets through the quark gluon plasma. The contribution is found to be largest at LHC energies, moderate at RHIC energies and negligible at SPS energies. Its detection will provide a further proof of the existence of the QGP as well as of multiple interactions suffered by the high energy partons eventually leading to jet-quenching.

\section{Acknowledgments}

This work was supported by DOE grants DE-FG0296ER40945 and DE-AC02-98CH10886 and the Natural Sciences and Engineering Research Council of Canada. RJF is supported by the Feodor Lynen program of the Alexander von Humboldt Foundation. We are indebted to Berndt Müller for valuable comments. 
[1] S. D. Drell and T. M. Yan, Phys. Rev. Lett. 25, 316 (1970).

[2] E. L. Feinberg, Nuovo Cimento A 34, 391 (1976); E. V. Shuryak, Phys. Lett. B 78, 150 (1978).

[3] See, for example, C. Gale, Nucl. Phys. A 698, 143 (2002), and references therein.

[4] R. Vogt, B. V. Jacak, P. L. McGaughey and P. V. Ruuskanen, Phys. Rev. D 49, 3345 (1994); S. Gavin, P. L. McGaughey, P. V. Ruuskanen and R. Vogt, Phys. Rev. C 54, 2606 (1996); D. Fein, Z. P. Valerio and I. Sarcevic, Phys. Rev. C 56, 1637 (1997).

[5] K. J. Eskola and J. Lindfors, Z. Phys. C 46, 141 (1990).

[6] K. Geiger and J. I. Kapusta, Phys. Rev. Lett. 70, 1920 (1993).

[7] B. Kämpfer and O. P. Pavlenko, Nucl. Phys. A 566, 351c (1994).

[8] M. Strickland, Phys. Lett. B 331, 245 (1994).

[9] D. K. Srivastava, M. G. Mustafa and B. Müller, Phys. Rev. C 56, 1064 (1997).

[10] See, for example, D. Adamova et al. [CERES Collaboration], Nucl. Phys. A 698, 253 (2002), and references therein.

[11] See, for example, R. Rapp and J. Wambach, Adv. Nucl. Phys. 25, 1 (2000), and references therein.

[12] M. Masera et al. [HELIOS Collaboration], Nucl. Phy. A 590, 93c (1995); M. C. Abreu et al. [NA50 Collaboration], Eur. Phy. J. C 14, 443 (2000).

[13] D. K. Srivastava, B. Sinha and C. Gale, Phys. Rev. C 53, 567 (1996).

[14] R. Rapp and E. Shuryak, Phys. Lett. B 473, 13 (2000).

[15] I. Kvasnikova, C. Gale and D. K. Srivastava, Phys. Rev. C 65, 064903 (2002).

[16] J. L. Nagle, for PHENIX Collaboration, nuclex/0209015

[17] M. Gyulassy and X. Wang, Nucl. Phys. B 420, 583 (1994); R. Baier, Y. L. Dokshitzer, A. H. Mueller, S. Peigne and D. Schiff, Nucl. Phys. B 484, 265 (1997).

[18] R. J. Fries, B. Müller and D. K. Srivastava, nuclth/0208001.

[19] Z. Lin and M. Gyulassy, Phys. Rev. C 51, 2177 (1995).

[20] J. D. Bjorken, Phys. Rev. D 27, 140 (1983).

[21] J. Kapusta, L. D. McLerran and D. K. Srivastava, Phys. Lett. B 283, 145 (1992).

[22] K. Kajantie, J. Kapusta, L. D. McLerran and A. Mekjian. Phys. Rev. D 34, 2746 (1986).

[23] R. C. Hwa and K. Kajantie, Phys. Rev. D 32, 1109
(1985)

[24] J. Alam, D. K. Srivastava, B. Sinha and D. N. Basu, Phys. Rev. D 48, 1117 (1993).

[25] K. Gallmeister, B. Kämpfer and O. P. Pavlenko, Phys. Rev. C 66, 014908 (2002).

[26] K. J. Eskola, B. Müller and X. Wang, Phys. Lett. B 374, 20 (1996)

[27] T. S. Biro, E. van Doorn, M. H. Thoma, B. Müller and X.-N. Wang, Phys. Rev. C 48, 1275 (1993).

[28] J. F. Owens, Rev. Mod. Phys. 59, 465 (1987).

[29] H. L. Lai et al. [CTEQ Collaboration], Eur. Phys. J. C 12, 375 (2000).

[30] K. J. Eskola, V. J. Kolhinen and C. A. Salgado, Eur. Phys. J. C 9, 61 (1999).

[31] R. Yoshida, T. Miyazaki, and M. Kadoy, Phys. Rev. D 35, 388 (1987); K. Redlich, Phys. Rev. D, 36 (1987); B. Datta, S. Raha, and B. Sinha, Nucl. Phys. A 490, 733 (1988); S. Hirasawa, M. Kadoya, and T. Miyazaki, Phys. Lett. B 218, 263 (1989); D. K. Srivastava, B. Sinha, and T. C. Awes, Phys. Lett. B 387, 21 (1996); M. Hentschel, B. Kämpfer, O. P. Pavlenko, K. Redlich, and G. Soff, Z. Phys. C 75, 333 (1997); S. Sarkar, D. K. Srivastava, B. Sinha, P. K. Roy, S. Chattopadhyay, and D. Pal, Phys. Lett. B 402, 13 (1997).

[32] R. J. Fries, C. Gale, D. K. Srivastava, to be published.

[33] G. G. Barnaföldi, G. Fai, P. Levai, G. Rapp, and Y. Zhang, J.Phys. G 27, 1767 (2001).

[34] S. Gavin and J. Milana, Phys. Rev. Lett. 68, 1834 (1992); R. Vogt, Phys. Rev. C 61, 035203 (2000).

[35] B. Svetitsky, Phys. Rev. D 37, 2484 (1988); M. G. Mustafa, D. Pal and D. K. Srivastava, Phys. Rev. C 57, 889 (1998); M. G. Mustafa, D. Pal, D. K. Srivastava and M. Thoma, Phys. Lett. B 428, 234 (1998).

[36] E. Shuryak, Phys. Rev. C 55, 961 (1997); Z. Lin, R. Vogt and X.-N. Wang, Phys. Rev. C 57, 899 (1998); B. Kämpfer and O. P. Pavlenko, Phys. Lett. B 391, 185 (1997).

[37] Yu. L. Dokshitzer and D. E. Kharzeev, Phys. Lett. B 519, 199 (2001); R. Baier, D. Schiff, and B. G. Zakharov, hep-ph/0002198.

[38] http://na60.web.cern.ch/NA60/

[39] K. J. Eskola and X.-N. Wang, Phys. Rev. D 49, 4542 (1994).

[40] S. A. Bass, B. Müller and D. K. Srivastava, nuclth/0207042 